\title{
Review \\ Multivalue Collocation Methods for Ordinary and Fractional Differential Equations
}

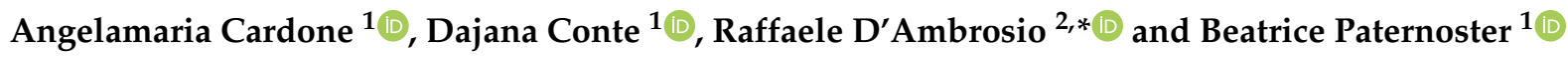 \\ 1 Department of Mathematics, University of Salerno, 84084 Fisciano, Italy; ancardone@unisa.it (A.C.); \\ dajconte@unisa.it (D.C.); beapat@unisa.it (B.P.) \\ 2 Department of Information Engineering and Computer Science and Mathematics, University of L'Aquila, \\ 67100 L'Aquila, Italy \\ * Correspondence: raffaele.dambrosio@univaq.it
}

check for

updates

Citation: Cardone, A.; Conte, D.;

D'Ambrosio, R.; Paternoster, P. Multivalue Collocation Methods for Ordinary and Fractional Differential Equations. Mathematics 2022, 10, 185. https://doi.org/10.3390/ math10020185

Academic Editors: Francesco Aldo Costabile, Maria I. Gualtieri and Anna Napoli

Received: 29 November 2021

Accepted: 4 January 2022

Published: 7 January 2022

Publisher's Note: MDPI stays neutral with regard to jurisdictional claims in published maps and institutional affiliations.

Copyright: (C) 2022 by the authors. Licensee MDPI, Basel, Switzerland. This article is an open access article distributed under the terms and conditions of the Creative Commons Attribution (CC BY) license (https:// creativecommons.org/licenses/by/ $4.0 /)$.

\begin{abstract}
The present paper illustrates some classes of multivalue methods for the numerical solution of ordinary and fractional differential equations. In particular, it focuses on two-step and mixed collocation methods, Nordsieck GLM collocation methods for ordinary differential equations, and on two-step spline collocation methods for fractional differential equations. The construction of the methods together with the convergence and stability analysis are reported and some numerical experiments are carried out to show the efficiency of the proposed methods.
\end{abstract}

Keywords: ordinary differential equations; fractional differential equations; multistep methods; collocation; convergence; stability

MSC: 65L05; 65L20; 26A33

\section{Introduction}

Numerical collocation is an effective technique for the approximation of solutions to a given functional equation by means of a continuous approximate belonging to a finite dimensional space spanned by functions chosen in accordance with the qualitative behavior of the exact solution. This idea has successfully been applied in several contexts (a very brief and far from being extensive, list of contributions in the existing literature can be found in [1-36] and references therein).

In this paper, we aim to collect some of our recent results showing the effectiveness of collocation in two selected cases:

- Firstly, the case of stiff differential problems [1-3,37,38], commonly arising from spatially discretized time-dependent partial differential equations. This problem commonly exposes numerical schemes to the order reduction phenomena, typically characterizing low stage-order methods such as Runge-Kutta methods on Gaussian collocation points [1]. It is worth highlighting that improving the numerics for stiff problems has a direct impact on the numerical treatment of a wide class of problems that is interesting in several applications. A relevant case is given, for instance, by multiscale problems: Quoting from [39], "Stiff equations are multiscale problems" and this situation typically characterizes coupled physical systems whose components vary on different time-scales. It is the case, for instance, of epidemiological models for influenza or pandemics (see, for instance, refs. [40-42] and references therein), since multiscale models are an ideal framework to simultaneously simulate several processes such as immune response, pharmacokinetics, and interactions between virus and host.

Our proposal to remove order reduction in providing approximate solutions to stiff problems is to employ multivalue numerical methods based on numerical collocation. 
These methods are free from order reduction, as it happens for classical collocation methods. This topic is the subject of Sections 2 and 3;

- Secondly, the case of fractional differential problems, representing a fundamental tool to model anomalous diffusion [43], material hereditariness, viscoelastic materials [44], and heat conduction [45]. For these problems, the analytical solution is generally not available and the numerical treatment is not an easy task, due to the lack of smoothness of the analytical solution and general methods for Ordinary Differential Equations (ODEs), applied to Fractional Differential Equations (FDEs), generally exhibit low order of convergence, e.g., predictor-corrector methods [46]. Therefore ad hoc numerical methods should be formulated to obtain a higher degree of accuracy, as for example fractional linear multistep methods [47], a class of product integration methods [48]. In this scenario, an important role is played by collocation methods, as for example B-spline wavelets collocation [28], Chebychev collocation [49], spectral collocation [16,33,50,51], and non-polynomial collocation [25]. In this paper, we focus on spline collocation methods, which were first introduced by Blank [52], however the main contribution to the development and analysis of these methods has been given in $[19,29,30,53]$. More recently, multivalue spline collocation methods have been proposed $[18,20,54]$. This topic is the subject of Section 4.

\section{Multivalue Collocation Methods}

Multivalue methods for the numerical solution of ODEs [1,37,55-57]:

$$
\left\{\begin{array}{l}
y^{\prime}(t)=f(y(t)), \quad t \in\left[t_{0}, T\right] \\
y\left(t_{0}\right)=y_{0}
\end{array}\right.
$$

with $y:\left[t_{0}, T\right] \rightarrow \mathbb{R}^{d} f: \mathbb{R}^{d} \rightarrow \mathbb{R}^{d}$, and have the form:

$$
\begin{array}{ll}
Y_{i}^{[n]}=h \sum_{j=1}^{m} a_{i j} f\left(Y_{j}^{[n]}\right)+\sum_{j=1}^{r} u_{i j} y_{j}^{[n]}, \quad i=1,2, \ldots, m, \\
y_{i}^{[n+1]}=h \sum_{j=1}^{m} b_{i j} f\left(Y_{j}^{[n]}\right)+\sum_{j=1}^{r} v_{i j} y_{j}^{[n]}, \quad i=1,2, \ldots, r,
\end{array}
$$

where $t_{n}=t_{0}+n h, n=0,1, \ldots, N$ are the grid points and $h=\left(T-t_{0}\right) / N$ is a fixed stepsize. The matrices:

$$
\mathbf{A}=\left[a_{i j}\right] \in \mathbb{R}^{m \times m}, \quad \mathbf{U}=\left[u_{i j}\right] \in \mathbb{R}^{m \times r}, \quad \mathbf{B}=\left[b_{i j}\right] \in \mathbb{R}^{r \times m}, \quad \mathbf{V}=\left[v_{i j}\right] \in \mathbb{R}^{r \times r}
$$

are the coefficients of the methods and the vector $\mathbf{c}=\left[c_{1}, c_{2}, \ldots, c_{m}\right]^{T}$ is called the abscissa vector. The parameters $c_{1}, c_{2}, \ldots, c_{m}$ are usually included in $[0,1]$, but in some cases can be taken outside this interval in order to obtain $A$-stability (see for example Figure 1).

The values $Y_{i}^{[n]} \in \mathbb{R}^{d}$ are called internal stages and provide an approximation to $y\left(t_{n}+c_{i} h\right)$, while $y_{i}^{[n]} \in \mathbb{R}^{d}$ are called external stages, and each $y_{i}^{[n]}$ provides an approximation to a linear combination of the derivatives of $y$ at the point $t_{n}$. The number of internal stages $m$ and the number of external stages $r$, influence the order of convergence and the computational cost of the method, as will be shown later for some classes of methods.

As usual, the coefficient matrices of the multivalue numerical method (2) can be gathered in the Butcher tableau:

$$
\left[\begin{array}{l|l}
\mathbf{A} & \mathbf{U} \\
\hline \mathbf{B} & \mathbf{V}
\end{array}\right]
$$


Collocation multivalue numerical methods represent a continuous extension of multivalue numerical methods in the GLM (General Linear Method) form (2), by means of following piecewise collocation polynomial:

$$
P_{n}\left(t_{n}+\theta h\right)=\sum_{i=1}^{r} \alpha_{i}(\theta) y_{i}^{[n]}+h \sum_{i=1}^{m} \beta_{i}(\theta) f\left(P_{n}\left(t_{n}+c_{i} h\right)\right), \quad \theta \in[0,1],
$$

and by interpreting the internal stages in (2) as $Y_{i}^{[n]}=P_{n}\left(t_{n}+c_{i} h\right)$. In (5), the polynomials $\alpha_{i}(\theta)$ and $\beta_{i}(\theta)$ have a degree equal to the order $p$ of the method and are usually computed by solving continuous order conditions, as will be described in the following.

Several kind of multivalue collocation methods have been introduced so far, with a different form for the vector of external stages. We will describe in the next two subsections two different choices which lead to two-step collocation methods and Nordsieck GLM collocation methods.

\subsection{Two-Step Collocation Methods}

Two-step collocation collocation methods have been introduced in [5] and have the form:

$$
\begin{aligned}
& Y_{i}^{[n]}=\varphi_{0}\left(c_{i}\right) y_{n-1}+\varphi_{1}\left(c_{i}\right) y_{n}+h \sum_{j=1}^{m}\left(\psi_{j}\left(c_{i}\right) f\left(Y_{j}^{[n]}\right)+\chi_{j}\left(c_{i}\right) f\left(Y_{j}^{[n-1]}\right)\right) \quad i=1,2, \ldots, m, \\
& y_{n+1}=\varphi_{0}(1) y_{n-1}+\varphi_{1}(1) y_{n}+h \sum_{j=1}^{m}\left(\psi_{j}(1) f\left(Y_{j}^{[n]}\right)+\chi_{j}(1) f\left(Y_{j}^{[n-1]}\right)\right) .
\end{aligned}
$$

with a collocation polynomial defined by:

$$
P_{n}\left(t_{n}+\theta h\right)=\varphi_{0}(\theta) y_{n-1}+\varphi_{1}(\theta) y_{n}+h \sum_{j=1}^{m}\left(\psi_{j}(\theta) f\left(P\left(t_{n}+c_{j} h\right)\right)+\chi_{j}(\theta) f\left(P\left(t_{n-1}+c_{j} h\right)\right)\right),
$$

with $\theta \in[0,1]$ and $Y_{j}^{[n]}=P\left(t_{n}+c_{j} h\right), Y_{j}^{[n-1]}=P\left(t_{n-1}+c_{j} h\right)$.

We observe that such methods can be viewed as multivalue collocation methods (2)-(5), by choosing $r=m+2$,

$$
\begin{gathered}
\alpha_{1}(\theta)=\varphi_{1}(\theta), \quad \alpha_{2}(\theta)=\varphi_{0}(\theta), \\
\alpha_{2+i}(\theta)=\chi_{i}(\theta), \quad \beta_{i}(\theta)=\psi_{i}(\theta) \quad i=1, \ldots, m
\end{gathered}
$$

and

$$
y^{[n]}=\left[\begin{array}{c}
y_{n} \\
y_{n-1} \\
h F\left(Y^{[n-1]}\right)
\end{array}\right] \in \mathbb{R}^{m+2},
$$

where,

$$
Y^{[n]}=\left[\begin{array}{c}
Y_{1}^{[n]} \\
\vdots \\
Y_{m}^{[n]}
\end{array}\right], \quad F\left(Y^{[n]}\right)=\left[\begin{array}{c}
f\left(Y_{1}^{[n]}\right) \\
\vdots \\
f\left(Y_{m}^{[n]}\right)
\end{array}\right] .
$$

With the choice (8) for the external approximation vector, the collocation polynomial (5) is a global smooth extension of the GLM (2) with tableau (4) given by the following matrices:

$$
\begin{aligned}
& \mathbf{A}=\left[\beta_{j}\left(c_{i}\right)\right]_{i, j=1, \ldots, m} \in \mathbb{R}^{m \times m}, \quad \mathbf{U}=\left[\alpha_{j}\left(c_{i}\right)\right]_{i=1, \ldots, m, j=1, \ldots, r} \in \mathbb{R}^{m \times r}, \\
& \mathbf{w}=\left[\alpha_{j}(1)\right]_{j=1, \ldots, r} \in \mathbb{R}^{r}, \quad \mathbf{v}=\left[\beta_{j}(1)\right]_{j=1, \ldots, m} \in \mathbb{R}^{m} \\
& \mathbf{B}=\left[\begin{array}{c}
\mathbf{v}^{T} \\
\mathbf{0} \\
\mathbf{I}
\end{array}\right] \in \mathbb{R}^{r \times m}, \quad \mathbf{V}=\left[\begin{array}{ccc}
\mathbf{w}^{T} \\
1 & 0 & \mathbf{0} \\
0 & 0 & \mathbf{0}
\end{array}\right] \in \mathbb{R}^{r \times r},
\end{aligned}
$$


where $\mathbf{I}$ is the identity matrix of dimension $m$ and $\mathbf{0}$ is a zero matrix or vector of suitable dimensions.

Order conditions can be formalized by the following theorem [5].

Theorem 1. A multivalue collocation method (2) with collocation polynomial in (5) and vector of external stages defined by (8) is an approximation of uniform order $p$ to the solution of the wellposed problem approximates in the solution of (1) with uniform order $p$, if the following conditions are satisfied:

$$
\begin{gathered}
\alpha_{1}(\theta)+\alpha_{2}(\theta)=1 \\
v !
\end{gathered}
$$

The maximum attainable order is $p=m+r-1$, as in this case of $m+r-1$ polynomials $\alpha_{i}(\theta)$ and $\beta_{i}(\theta)$, which are uniquely derived by solving the continuous order conditions (10), and the corresponding collocation polynomial satisfies the conditions listed in the following corollary [5].

Corollary 1. The maximum attainable uniform order of convergence for a multivalue collocation method (2) with collocation polynomial in (5) and vector of external stages defined by (8) is $p=2 m+1=m+r-1$. The corresponding collocation polynomial satisfies the following interpolation and collocation conditions:

$$
P_{n}\left(t_{n}\right)=y_{n}, \quad P_{n}\left(t_{n-1}\right)=y_{n-1},
$$

$P_{n}^{\prime}\left(t_{n}+c_{i} h\right)=f\left(P_{n}\left(t_{n}+c_{i} h\right)\right), \quad P_{n}^{\prime}\left(t_{n-1}+c_{i} h\right)=f\left(P_{n}\left(t_{n-1}+c_{i} h\right)\right), \quad i=1,2, \ldots, m$.

\subsection{Nordsieck GLM Collocation Methods}

Nordsieck GLM collocation methods have been introduced in [4] and rely on the vector of external stages in the so-called Nordsieck form [37]:

$$
y^{[n]}=\left[\begin{array}{c}
y_{1}^{[n]} \\
y_{2}^{[n]} \\
\vdots \\
y_{r}^{[n]}
\end{array}\right] \approx\left[\begin{array}{c}
y\left(t_{n}\right) \\
h y^{\prime}\left(t_{n}\right) \\
\vdots \\
h^{r-1} y^{r-1}\left(t_{n}\right) .
\end{array}\right]
$$

With this choice for an external approximation vector, the collocation polynomial (5) is a global smooth extension of the GLM (2) with tableau (4) given by the following matrices:

$$
\begin{aligned}
& \mathbf{A}=\left[\beta_{j}\left(c_{i}\right)\right]_{i, j=1, \ldots, m} \in \mathbb{R}^{m \times m}, \mathbf{U}=\left[\alpha_{j}\left(c_{i}\right)\right]_{i=1, \ldots, m, j=1, \ldots, r} \in \mathbb{R}^{m \times r}, \\
& \mathbf{B}=\left[\beta_{j}^{(i-1)}(1)\right]_{i=1, \ldots, m, j=1, \ldots, r} \in \mathbb{R}^{r \times m}, \mathbf{V}=\left[\alpha_{j}^{(i-1)}(1)\right]_{i, j=1, \ldots, r} \in \mathbb{R}^{r \times r},
\end{aligned}
$$

Order conditions have been derived in [4], as stated in the following theorem.

Theorem 2. A multivalue collocation method (2) with collocation polynomial in (5) and vector of external stages defined by (13) is an approximation of uniform order $p$ to the solution of the well-posed problem approximates of the solution of (1) with uniform order $p$, if and only if the following conditions are satisfied: 


$$
\begin{gathered}
\alpha_{1}(\theta)=1 \\
\frac{\theta^{v}}{v !}-\alpha_{v+1}(\theta)-\sum_{i=1}^{m} \frac{c_{i}^{v-1}}{(v-1) !} \beta_{i}(\theta)=0, \quad v=1, \ldots, r-1, \\
\frac{\theta^{v}}{v !}-\sum_{i=1}^{m} \frac{c_{i}^{v-1}}{(v-1) !} \beta_{i}(\theta)=0, \quad v=r, \ldots, p .
\end{gathered}
$$

Corollary 2. The maximum attainable uniform order of convergence for a multivalue collocation method (2) with a collocation polynomial in (5) and vector of external stages defined by (13) is $m+r-1$. The corresponding collocation polynomial satisfies the following interpolation and collocation conditions:

$$
\begin{gathered}
P_{n}\left(t_{n}\right)=y_{1}^{[n]}, \quad P_{n}^{\prime}\left(t_{n}\right)=y_{2}^{[n]}, \quad \ldots \quad P_{n}^{(r-1)}\left(t_{n}\right)=y_{r-1}^{[n]}, \\
P_{n}^{\prime}\left(t_{n}+c_{i} h\right)=f\left(P_{n}\left(t_{n}+c_{i} h\right)\right), \quad i=1,2, \ldots, m .
\end{gathered}
$$

\subsection{Derivation of A-Stable Multivalue Collocation Methods}

We describe in this section the existing procedures for the derivation of $A$-stable uniform order multivalue collocation methods. The advantages of deriving such methods lies in their efficiency in the numerical treatment of stiff problem, as they do not suffer from the order reduction phenomenon [1,2]. We recall that a numerical method is $A$-stable if its region of absolute stability includes the entire complex half-plane with a negative real part.

As we observe from Corollaries 1 and 2, the maximum attainable uniform order multivalue collocation methods with collocation polynomial in (5) and vector of external stages defined by (8) or (13) is $p=m+r-1$. With the aim of deriving $A$-stable methods, according to the Daniel-Moore theorem [1], the order of the method cannot exceed $2 \mathrm{~m}$. Therefore the following Theorem clarifies the restriction, on the number of external stages, necessary for $A$-stability. The proof can be found in [4].

Theorem 3. An A-stable multivalue collocation method with collocation polynomial in (5) fulfills the constraint $r \leq m+1$.

As a consequence, two-step collocation methods of Section 2.1 cannot be $A$-stable, as for these methods $r=m+2$, while $A$-stable Nordsieck GLM collocation methods of Section 2.2 can be derived with a suitable choice for $r$.

In regards to two-step collocation methods, in the paper [5], $A$-stable methods of uniform order $p=m+s, s=1,2, \ldots, m$ have been derived by imposing not all the order conditions up to $p=2 m+1$, but just requiring the fulfillment of the first $m+s$ order conditions in (10). This procedure corresponds to relaxing some of the interpolation/collocation conditions in (11) and (12), and the corresponding methods are called two-step almost collocation methods.

In regards to Nordsieck GLM collocation methods, in the paper [4], $A$-stable methods of uniform order $p=m+r-1$ with $r=m+1$ have been provided.

Regarding the computational cost of multivalue collocation methods (2)-(5), it is strongly related to the solution of the nonlinear system for the computation of the vector $Y^{[n]}$ in (2), and depends on the matrix $\mathbf{A}=\left[\beta_{j}\left(c_{i}\right)\right]_{i, j=1, \ldots, m}$. Two-step almost collocation methods having lower triangular or diagonal coefficient matrix $\mathbf{A}$ that have been derived in [24]. Regarding Nordsieck GLM collocation methods, the requirement for a structured coefficient matrix forces the relaxation of some of the interpolation/collocation conditions (15) and (16), thus leading to Nordsieck GLM almost collocation methods with $r=m+1$, having order $p=r$ or $p=r-1$, i.e., obtained by imposing not all the order conditions up to $p=m+r-1$, but just requiring the fulfillment of the first $r$ or $r-1$ order conditions in (14). 
We now provide examples of $A$-stable multivalue collocation and almost collocation methods belonging to the class described in Section 2.2. We consider the case of $m=2$ and $r=m+1=3$. The collocation polynomial assumes the form:

$$
\begin{aligned}
P_{n}\left(t_{n}\right. & +\vartheta h)=y_{1}^{[n]}+\alpha_{2}(\vartheta) y_{2}^{[n]}+\alpha_{3}(\vartheta) y_{3}^{[n]} \\
& +h\left(\beta_{1}(\vartheta) f\left(P\left(t_{n}+c_{1} h\right)\right)+\beta_{2}(\vartheta) f\left(P\left(t_{n}+c_{2} h\right)\right)\right)
\end{aligned}
$$

and the corresponding Butcher tableau is given by:

$$
\left[\begin{array}{c|c}
\mathbf{A} & \mathbf{U} \\
\hline \mathbf{B} & \mathbf{V}
\end{array}\right]=\left[\begin{array}{cc|ccc}
\beta_{1}\left(c_{1}\right) & 0 & 1 & \alpha_{2}\left(c_{1}\right) & \alpha_{3}\left(c_{1}\right) \\
\beta_{1}\left(c_{2}\right) & \beta_{2}\left(c_{2}\right) & 1 & \alpha_{2}\left(c_{2}\right) & \alpha_{3}\left(c_{2}\right) \\
\hline \beta_{1}(1) & \beta_{2}(1) & 1 & \alpha_{2}(1) & \alpha_{3}(1) \\
\beta_{1}^{\prime}(1) & \beta_{2}^{\prime}(1) & 0 & \alpha_{2}^{\prime}(1) & \alpha_{3}^{\prime}(1) \\
\beta_{1}^{\prime \prime}(1) & \beta_{2}^{\prime \prime}(1) & 0 & \alpha_{2}^{\prime \prime}(1) & \alpha_{3}^{\prime \prime}(1)
\end{array}\right] .
$$

We consider the following forms for the matrix $\mathbf{A}$ :

- $\quad$ Full matrix [4] (GLM-F);

- Lower triangular matrix (GLM-T);

- Singly lower triangular matrix (GLM-S);

- Diagonal matrix (GLM-D).

Polynomials $\alpha_{j}(\theta)$ and $\beta_{j}(\theta)$ in (17) are constructed by imposing order conditions of Theorem 2 with $p=4$ in the case of GLM-F and $p=3$ in the case of GLM-T, GLM-S, and GLM-D. Figure 1 shows the region of $A$-stability in the $\left(c_{1}, c_{2}\right)$ plane for all the classes of methods.

Examples of A-stable methods have the following Butcher tableau:

- GLM-F:

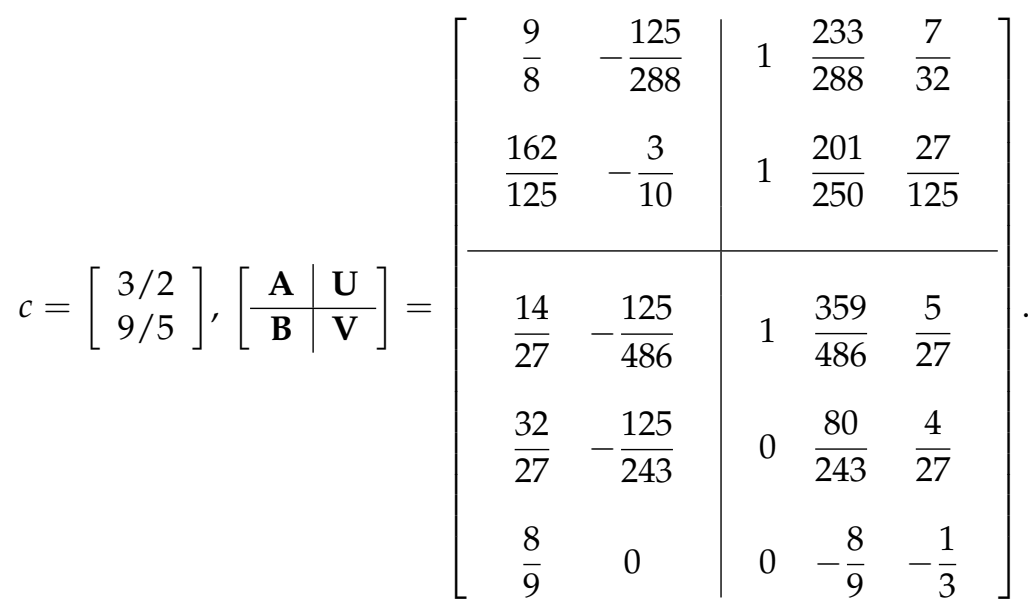

- GLM-T: 


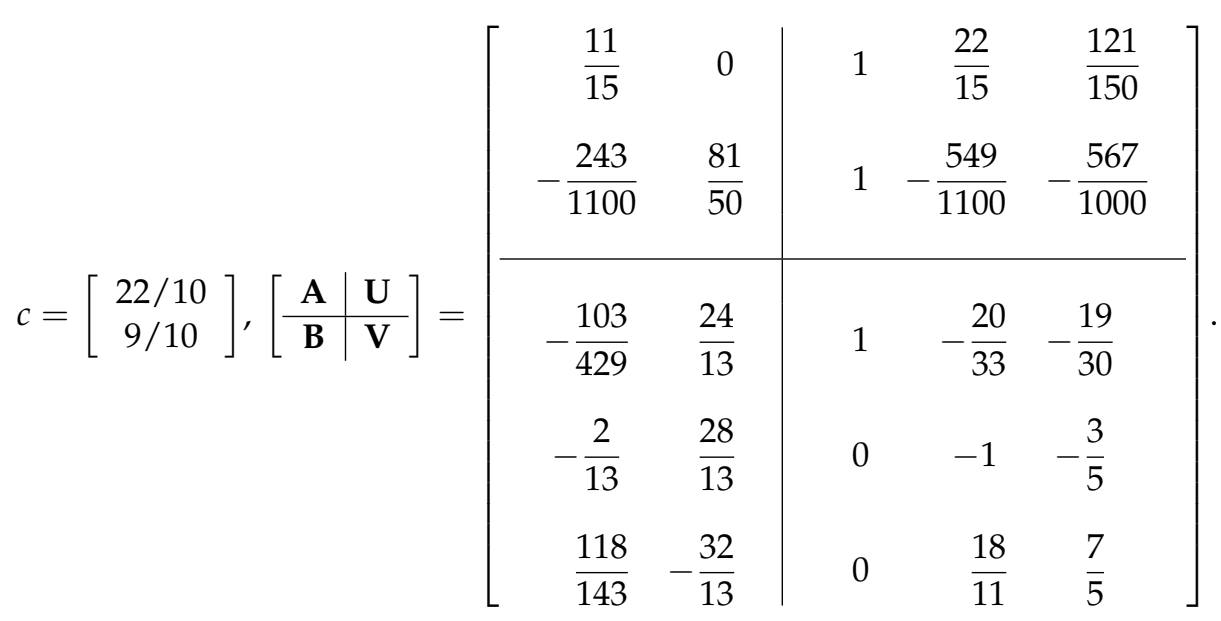

- GLM-S:

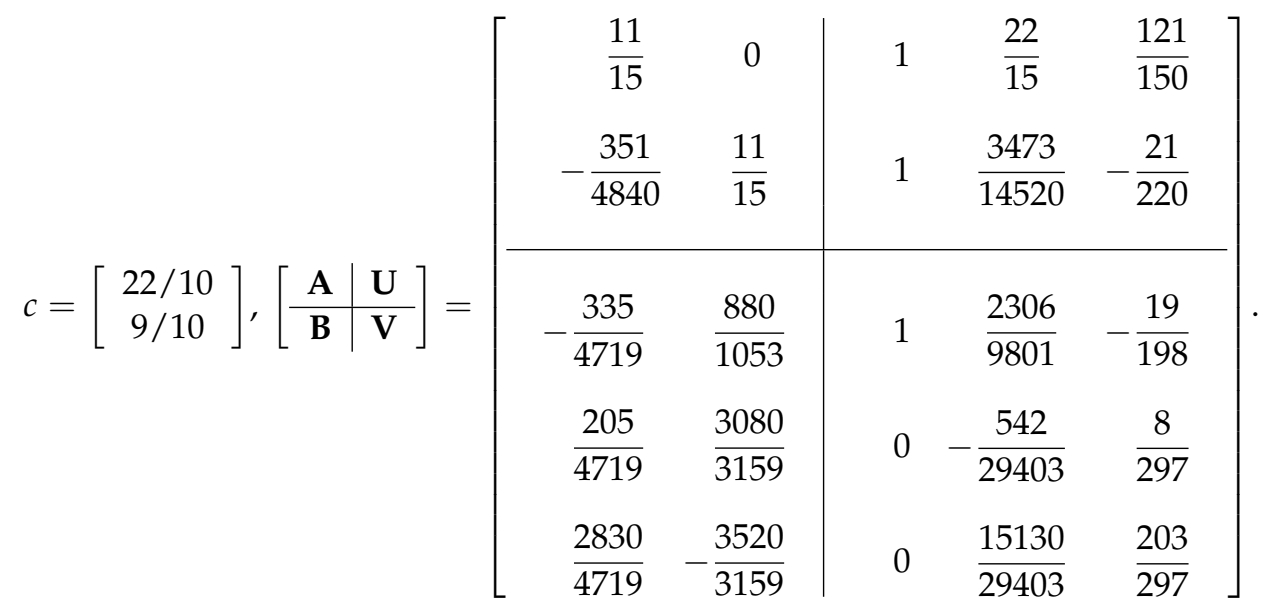

- GLM-D:

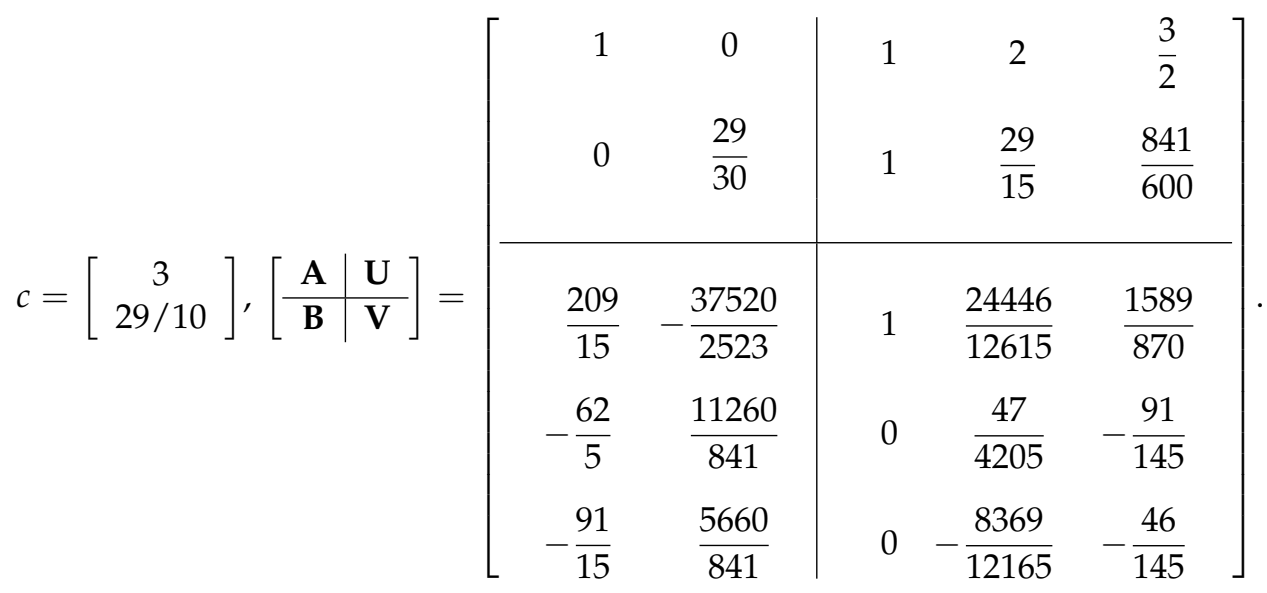



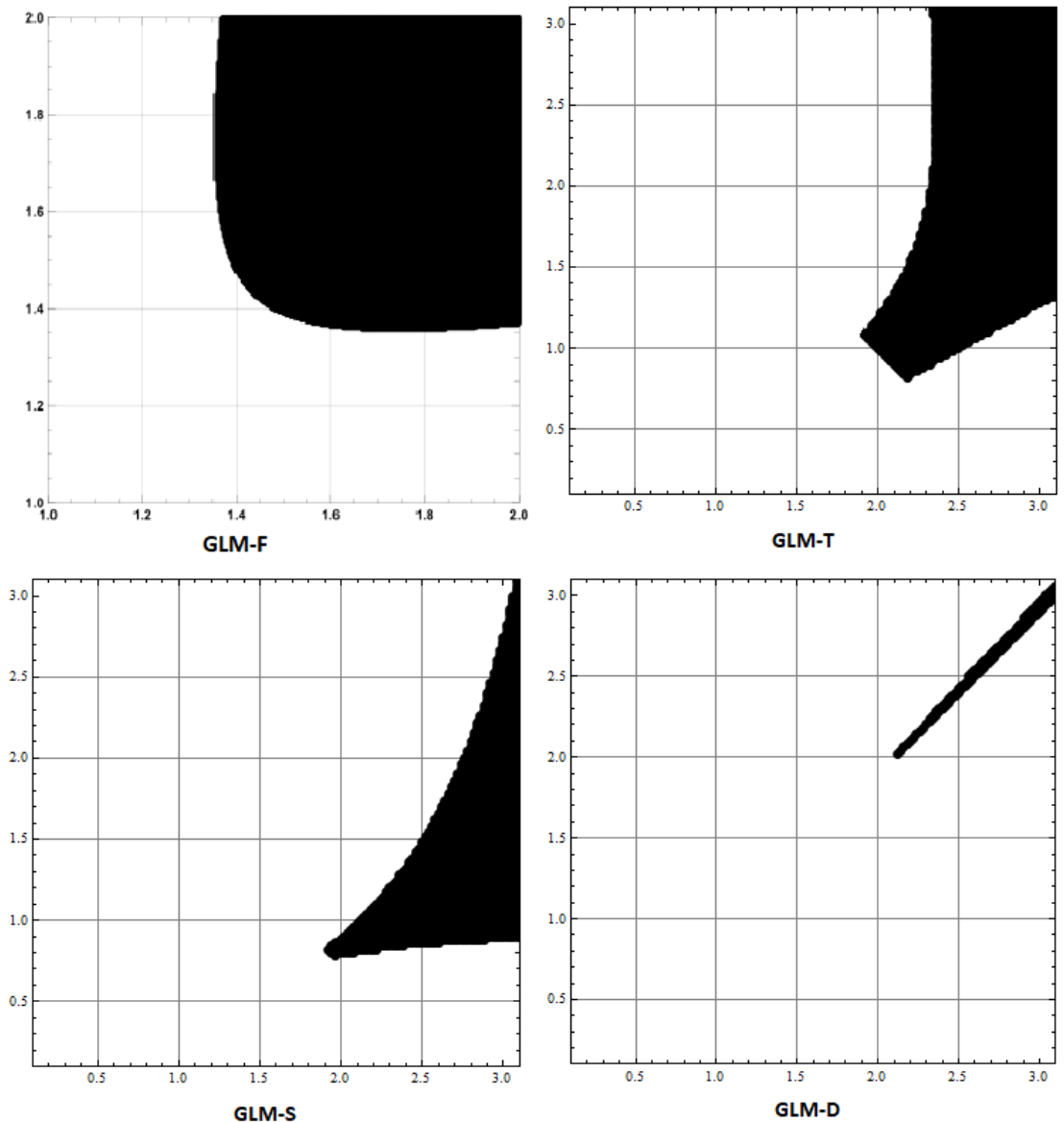

Figure 1. Regions of A-stability in the $\left(c_{1}, c_{2}\right)$ plane.

\subsection{Numerical Illustration}

We now show the behavior of the methods listed in the previous section on the Prothero-Robinson problem [1,2]:

$$
\left\{\begin{array}{l}
y^{\prime}(t)=\lambda(y(t)-\sin (t))+\cos (t), \quad t \in[0,10], \\
y(0)=0
\end{array}\right.
$$

which is stiff when $\lambda \ll 0$. We compare the results of the aforementioned methods with those obtained by the two-stage Gaussian Runge-Kutta (RK) method. We report in Table 1 the results obtained for $\lambda=-10^{6}$ in (18), by applying multivalue collocation and almost collocation methods together with the Runge-Kutta (RK) method of Gauss:

$$
\begin{array}{c|cc}
\frac{1}{2}-\frac{\sqrt{3}}{6} & \frac{1}{4} & \frac{1}{4}-\frac{\sqrt{3}}{6} \\
\frac{1}{2}+\frac{\sqrt{3}}{6} & \frac{1}{4}+\frac{\sqrt{3}}{6} & \frac{1}{4} \\
\hline & \frac{1}{2} & \frac{1}{2}
\end{array}
$$

The method (19), which has order 4 and uniform order 2, therefore suffers from order reduction when applied to a stiff problem. Table 1 shows the error in the final step point for different values of the step size and the experimental order of the methods for $\lambda=-10^{6}$. We observe that the Runge-Kutta method exhibits order reduction, while this is not the case for multivalue collocation and almost collocation methods, having order 4 but uniform order 2 , hence it suffers from order reduction on stiff problems, as is visible from 
Table 1. Multivalue collocation and almost collocation methods, instead, keep their order of convergence.

Table 1. Absolute errors in the final step point and experimental orders of convergence for problem (18) with $\lambda=-10^{6}$.

\begin{tabular}{|c|c|c|c|c|c|c|c|c|c|c|}
\hline \multirow[t]{2}{*}{$\mathbf{h}$} & \multicolumn{2}{|l|}{ GLM-F } & \multicolumn{2}{|l|}{ GLM-T } & \multicolumn{2}{|l|}{ GLM-S } & \multicolumn{2}{|l|}{ GLM-D } & \multicolumn{2}{|l|}{ RK } \\
\hline & Error & $p$ & Error & $p$ & Error & $p$ & Error & $p$ & Error & $p$ \\
\hline $1 / 10$ & $2.41 \times 10^{-8}$ & & $1.51 \times 10^{-7}$ & & $1.51 \times 10^{-7}$ & & $4.88 \times 10^{-5}$ & & $1.52 \times 10^{-4}$ & \\
\hline $1 / 20$ & $7.50 \times 10^{-10}$ & 5.01 & $9.21 \times 10^{-9}$ & 4.03 & $9.21 \times 10^{-9}$ & 4.03 & $3.04 \times 10^{-6}$ & 4.01 & $3.84 \times 10^{-5}$ & 1.98 \\
\hline $1 / 40$ & $2.21 \times 10^{-11}$ & 5.08 & $5.69 \times 10^{-10}$ & 4.02 & $5.70 \times 10^{-10}$ & 4.01 & $1.89 \times 10^{-7}$ & 4.01 & $9.99 \times 10^{-6}$ & 1.94 \\
\hline $1 / 80$ & $7.06 \times 10^{-13}$ & 4.97 & $3.45 \times 10^{-11}$ & 4.04 & $3.58 \times 10^{-11}$ & 3.99 & $1.18 \times 10^{-8}$ & 4.00 & $2.78 \times 10^{-6}$ & 1.85 \\
\hline
\end{tabular}

\section{Multivalue Mixed Collocation Methods}

In this Section we describe the derivation of Nordsieck GLM mixed collocation methods of the form (2)-(5), with the vector of external stages in Nordsieck form (13). The idea is, instead of considering a basis of polynomials $\left\{\alpha_{i}(\theta), \beta_{j}(\theta), i=1, \ldots, r, j=1, \ldots, m\right\}$, to consider a basis constituted by a combination of trigonometric and polynomial functions. Such methods are useful for problems of the form (1) for which the exact solution is oscillatory with a known frequency of oscillation $\omega$.

As a consequence of Theorem 2, the polynomials $\alpha_{i}(\theta)$ and $\beta_{j}(\theta)$, associated to Nordsieck GLM collocation methods of uniform order $p=m+r-1$, have a degree of at most $m+r-1$. Therefore, they can be written in the form:

$$
\begin{array}{lr}
\alpha_{1}(\theta)=1 \quad \alpha_{i+1}(\theta)=\sum_{j=1}^{m+r-1} \frac{\bar{\mu}_{i, j-1}}{j} \theta^{j}, & i=1, \ldots, r-1, \\
\beta_{i}(\theta)=\sum_{j=1}^{m+r-1} \frac{\mu_{i, j-1}}{j} \theta^{j}, & i=1, \ldots, m .
\end{array}
$$

The idea of Nordsieck GLM mixed collocation methods introduced in [35] relies on considering new basis functions:

$$
\left\{\alpha_{i}^{T}(\theta, z), \beta_{j}^{T}(\theta, z), i=1, \ldots, r, j=1, \ldots, m\right\},
$$

which depend also on the frequency of oscillation of the problem, i.e., depending on $z=\omega h$, of the form:

$$
\begin{aligned}
\alpha_{1}^{T}(\theta, z) & =1 \\
\alpha_{i+1}^{T}(\theta, z) & =\frac{\bar{a}_{i}}{z} \sin (z \theta)-\frac{\bar{b}_{i}}{z} \cos (z \theta)+\frac{\bar{b}_{i}}{z}+\sum_{j=1}^{m+r-3} \frac{\bar{\gamma}_{i, j-1}}{j} \theta^{j} \quad i=1, \ldots, r-1, \\
\beta_{i}^{T}(\theta, z) & =\frac{a_{i}}{z} \sin (z \theta)-\frac{b_{i}}{z} \cos (z \theta)+\frac{b_{i}}{z}+\sum_{j=1}^{m+r-3} \frac{\gamma_{i, j-1}}{j} \theta^{j} \quad i=1, \ldots, m .
\end{aligned}
$$

The next theorem shows the expressions of coefficients $a_{i}, b_{i}, \gamma_{i, j}$ in (23) and $\bar{a}_{i}, \bar{b}_{i}, \bar{\gamma}_{i, j}$ in (24) in order to obtain the maximum attainable uniform order $p=m+r-1$. The proof can be found in [35].

Theorem 4. A multivalue mixed collocation method (2)-(5), with vector of external stages defined by (13) and functional basis $\left\{\alpha_{i}(\theta), \beta_{j}(\theta), i=1, \ldots, r, j=1, \ldots, m\right\}$ defined in (22)-(24), has order $p=m+r-1$ if:

$$
a_{i}=\frac{\operatorname{det} M_{i, 1}}{\operatorname{det} M}, \quad b_{i}=\frac{\operatorname{det} M_{i, 2}}{\operatorname{det} M}, \quad i=1, \ldots, m,
$$




$$
\begin{aligned}
& \gamma_{i j}=\left\{\begin{array}{ll}
(-1)^{\frac{j}{2}+1} a_{i} \frac{z^{j}}{j !} \quad j \text { is even, } & \\
(-1)^{\frac{j+1}{2}} b_{i} \frac{z^{j}}{j !} \quad j \text { is odd }, & j=0, \ldots, r-2, \\
\frac{\operatorname{det} M_{i, j-r+4}}{\operatorname{det} M}, & j=r-1, \ldots, m+r-4,
\end{array}, i=1, \ldots, m,\right. \\
& \bar{a}_{i}=\frac{\sum_{k=1}^{m}(-1)^{k+1} \frac{c_{k}^{i-1}}{(i-1) !} \operatorname{det} M_{k, 1}}{\operatorname{det} M}, \quad \bar{b}_{i}=\frac{\sum_{k=1}^{m}(-1)^{k} \frac{c_{k}^{i-1}}{(i-1) !} \operatorname{det} M_{k, 2}}{\operatorname{det} M}, \quad i=1, \ldots, r-1, \\
& \bar{\gamma}_{i j}= \begin{cases}(-1)^{\frac{j}{2}+1} \bar{a}_{i} \frac{z^{j}+\delta_{i, j+1}}{j !} \quad j \text { is even, } & j=0, \ldots, r-2, \\
(-1)^{\frac{j+1}{2}} \bar{b}_{i} \frac{z^{j}+\delta_{i, j+1}}{j !} j \text { is odd, } & \\
\sum_{k=1}^{m}(-1)^{k+j-r+1} \frac{c_{k}^{i-1}}{(i-1) !} \operatorname{det} M_{k, j-r+4} & j=r-1, \ldots, m+r-4,\end{cases}
\end{aligned}
$$$$
\text { where } M \text { is a square non singular matrix of order } m \text { given by: }
$$$$
M=\left(\begin{array}{cccccc}
\cos \left(z c_{1}\right)-\sum_{k=0}^{r_{1}}(-1)^{k} \frac{\left(z c_{1}\right)^{2 k}}{(2 k) !} & \sin \left(z c_{1}\right)-\sum_{k=0}^{r_{2}}(-1)^{k} \frac{\left(z c_{1}\right)^{2 k+1}}{(2 k+1) !} & c_{1}^{r-1} & c_{1}^{r} & \ldots & c_{1}^{m+r-4} \\
\cos \left(z c_{2}\right)-\sum_{k=0}^{r_{1}}(-1)^{k} \frac{\left(z c_{2}\right)^{2 k}}{(2 k) !} & \sin \left(z c_{2}\right)-\sum_{k=0}^{r_{2}}(-1)^{k} \frac{\left(z c_{2}\right)^{2 k+1}}{(2 k+1) !} & c_{2}^{r-1} & c_{2}^{r} & \ldots & c_{2}^{m+r-4} \\
\vdots & \vdots & \vdots & \vdots & \vdots & \vdots \\
\cos \left(z c_{m}\right)-\sum_{k=0}^{r_{1}}(-1)^{k} \frac{\left(z c_{m}\right)^{2 k}}{(2 k) !} & \sin \left(z c_{m}\right)-\sum_{k=0}^{r_{2}}(-1)^{k} \frac{\left(z c_{m}\right)^{2 k+1}}{(2 k+1) !} & c_{m}^{r-1} & c_{m}^{r} & \ldots & c_{m}^{m+r-4}
\end{array}\right),
$$

$M_{i, j}$ is the submatrix obtained by deleting the $i$-th row and $j$-th column from matrix $M, c_{1}, \ldots, c_{m}$ are the collocation points, $\delta_{i j}$ is the usual Kronecker delta, and:

$$
r_{1}=\left\{\begin{array}{ll}
\frac{r-2}{2} & r \text { is even, } \\
\frac{r-3}{2} & r \text { is odd },
\end{array} \quad r_{2}= \begin{cases}\frac{r-2}{2}-1 & r \text { is even, } \\
\frac{r-3}{2} & r \text { is odd } .\end{cases}\right.
$$

In order to show the performance of Nordsieck GLM mixed collocation methods, we show the results obtained on the following test problems:

- Problem 1:

$$
\left\{\begin{array}{l}
y^{\prime}(t)=-(y(t)-\sin (\omega t))+\omega \cos (\omega t), \quad t \in[0,10] \\
y(0)=0
\end{array}\right.
$$

whose solution is $y(t)=\sin (\omega t)$, so it is a function belonging to the basis.

- $\quad$ Problem 2:

$$
\left\{\begin{array}{l}
y^{\prime}(t)=-(y(t)-\sin (\omega t+t))+(\omega+1) \cos (\omega t+t), \quad t \in[0,10] \\
y(0)=0
\end{array}\right.
$$

whose solution is $y(t)=\sin (\omega t+t)=\sin \omega t \cos t+\cos \omega t \sin t$, so it is a combination of the basic functions $\sin \omega t$ and $\cos \omega t$.

We put $m=2$ and $r=3$ and $c_{1}=3 / 2, c_{2}=9 / 5$ and we denote by MGLM-F, the corresponding Nordsieck GLM mixed collocation method. In Table 2, we consider for a comparison, the GLM-F method of Section 2.3. The table clearly shows the advantage of mixed collocation versus polynomial collocation in the case of the oscillatory solution. 
Table 2. Absolute errors (in the final step point) and effective orders of convergence. Absolute errors in the final step point and experimental orders of convergence with $\omega=100$.

\begin{tabular}{lllllll}
\hline$h$ & GLM-F on Problem (29) & MGLM-F on Problem (29) & GLM-F on Problem (30) & MGLM-F on Problem (30) \\
\hline & Error & Error & Error & $p$ & Error & $p$ \\
\hline $1 / 40$ & 0.2764 & $1.4818 \times 10^{-12}$ & 0.3626 & & 0.0133 & \\
\hline $1 / 80$ & 0.0326 & $8.4277 \times 10^{-13}$ & 0.0436 & 3.0560 & $9.5762 \times 10^{-4}$ & 3.7958 \\
\hline $1 / 160$ & 0.0024 & $1.2212 \times 10^{-15}$ & 0.0031 & 3.8140 & $6.1966 \times 10^{-5}$ & 3.9499 \\
\hline $1 / 320$ & $1.5567 \times 10^{-4}$ & $8.9595 \times 10^{-14}$ & $1.9729 \times 10^{-4}$ & 3.9739 & $3.9064 \times 10^{-6}$ & 3.9876 \\
\hline $1 / 640$ & $9.8558 \times 10^{-6}$ & $1.4988 \times 10^{-14}$ & $1.2403 \times 10^{-5}$ & 3.9916 & $2.4468 \times 10^{-7}$ & 3.9969 \\
\hline
\end{tabular}

\section{Multivalue Spline Collocation Methods for FDEs}

In this section, we review multivalue spline collocation methods [18,20,54], applied to the IVP problem of type:

$$
\begin{cases}D^{\alpha} y(t)=f(t, y(t)), & t \in[0, b] \\ y^{(i)}(0)=\gamma_{i}, & i=0, \ldots, n-1,\end{cases}
$$

where $n-1<\alpha<n, n \in \mathbb{N}, \gamma_{i} \in \mathbb{R}, f:[0, b] \times \mathbb{R} \rightarrow R$. Here we consider the Caputo fractional derivative [58-60]:

$$
D^{\alpha} y(t)=\frac{1}{\Gamma(n-\alpha)} \int_{0}^{t} \frac{y^{(n)}(s)}{(t-s)^{\alpha+1-n}} d s .
$$

The convergence analysis of spline collocation methods is carried out in the functional space $C^{q, v}(0, T]$, defined as follows [15].

Let $q \in \mathbb{N}$ and $v \in(-\infty, 1)$, then $y:[0, T] \rightarrow \mathbb{R}$ belongs to the space $C^{q, v}(0, T]$ if it is $q$ times continuously differentiable in $(0, T]$, and:

$$
\left|y^{(i)}(t)\right| \leq c \begin{cases}1 & \text { if } i<1-v \\ 1+|\log t| & \text { if } i=1-v, t \in(0, T], i=1, \ldots, q \\ t^{1-v-i} & \text { if } i>1-v\end{cases}
$$

Sufficient conditions for obtaining a solution in the space $C^{q, v}(0, T]$ are provided by the following theorem.

Theorem 5 ([30]). Let $f \in C([0, T] \times \mathbb{R})$, q times continuously differentiable in $(0, T] \times \mathbb{R}$, and $\exists v \in[1-\alpha, 1)$ such that:

$$
\left|\frac{\partial^{i+j}}{\partial t^{i} \partial y^{j}} f(t, y)\right| \leq \phi(|y|)\left\{\begin{array}{ll}
1 & \text { if } i<1-v \\
1+|\log t| & \text { if } i=1-v, \\
t^{1-v-i} & \text { if } i>1-v
\end{array},(t, y) \in(0, T] \times \mathbb{R},\right.
$$

$\forall i, j \in \mathbb{N}$ with $i+j \leq q$. In addition, for $\alpha \in(0,1)$ assume that:

$$
\left|\frac{\partial^{i+j}}{\partial t^{i} \partial y^{j}}\left[f\left(t, y_{1}\right)-f\left(t, y_{2}\right)\right]\right| \leq \phi\left(\max \left\{\left|y_{1}\right|,\left|y_{2}\right|\right\}\right)\left|y_{1}-y_{2}\right|\left\{\begin{array}{ll}
1 & \text { if } i=0 \\
t^{1-v-i} & \text { if } i>0
\end{array},\right.
$$

$\left(t, y_{i}\right) \in(0, T] \times \mathbb{R}, i=1,2$. The function $\phi:[0, \infty) \rightarrow \mathbb{R}$ is assumed to be monotonically increasing. Let the fractional IVP (31) have a solution $y \in C[0, T]$ with $D^{\alpha} y \in C[0, T]$. Then $y \in C^{q, v}(0, T]$ and $D^{\alpha} y \in C^{q, v}(0, T]$.

By setting $z=D^{\alpha} y$, we have:

$$
y=J^{\alpha} z+Q,
$$


with,

$$
\begin{aligned}
\left(J^{\alpha} z\right)(t) & =\frac{1}{\Gamma(\alpha)} \int_{0}^{t}(t-s)^{\alpha-1} z(s) d s, \quad t>0, \\
Q(t) & =\sum_{i=0}^{\lceil\alpha\rceil-1} \frac{\gamma_{i}}{i !} t^{i},
\end{aligned}
$$

$\lceil\alpha\rceil$ being the smallest integer not less than $\alpha$. With this position, we may rewrite the IVP (31) as a nonlinear equation:

$$
z=f\left(t, J^{\alpha} z+Q\right) .
$$

Once (35) is solved, $y$ can be computed by (32).

\subsection{One-Step Collocation Methods for FDEs}

Let us introduce a graded mesh $I_{N}$ on $[0, T]$ with grading exponent $r \geq 1$ :

$$
t_{j}=b\left(\frac{j}{N}\right)^{r}
$$

and collocation parameters $0 \leq \eta_{1}<\cdots<\eta_{m} \leq 1$. Let,

$$
S_{k}^{(-1)}\left(I_{N}\right)=\left\{v:\left.v\right|_{\left[t_{j-1}, t_{j}\right]} \in \Pi_{k}, j=1, \ldots, N\right\} .
$$

The one step collocation method approximates the solution $z$ of (35) by a function $v \in S_{m-1}^{(-1)}\left(I_{N}\right)$. The collocation solution $v$ is computed by imposing these collocation conditions, for $j=1, \ldots, N$ :

$$
v_{j}\left(t_{j k}\right)=f\left(t_{j k},\left(J^{\alpha} v\right)\left(t_{j k}\right)+Q\left(t_{j k}\right)\right), \quad k=1, \ldots, m .
$$

where $v_{j}:=\left.v\right|_{\left[t_{j-1}, t_{j}\right]}$. Then, the approximate solution of (31) is the function $y_{N}$, defined as:

$$
y_{N}=J^{\alpha} v+Q .
$$

Collocation conditions (38) give rise to the nonlinear system in the unknowns $z_{j k}:=v_{j}\left(t_{j k}\right):$

$$
z_{j k}=f\left(t_{j k}, \sum_{\mu=1}^{m} z_{j \mu}\left(J^{\alpha} \varphi_{j, \mu}\right)\left(t_{j k}\right)+\sum_{\lambda=1}^{j-1} \sum_{\mu=1}^{m} z_{\lambda \mu}\left(J^{\alpha} \varphi_{\lambda, \mu}\right)\left(t_{j k}\right)+Q\left(t_{j k}\right)\right), \quad k=1, \ldots, m,
$$

where $\varphi_{\lambda, \mu}$ is equal to the $\mu$-th Lagrange fundamental polynomial corresponding to the nodes $t_{\lambda 1}, \ldots, t_{\lambda m}$ in $\left[t_{\lambda-1}, t_{\lambda}\right]$, and it is null outside this interval.

The error is analyzed in the following theorem, where this quantity is used:

$$
E_{N}(p, v, r)= \begin{cases}N^{-r(1-v)} & \text { if } 1 \leq r \leq \frac{p}{1-v} \\ N^{-p}(1+\log N) & \text { if } r=\frac{p}{1-v}=1 \\ N^{-p} & \text { if } r=\frac{p}{1-v}>1 \text { or } r>\frac{p}{1-v} .\end{cases}
$$

Theorem 6 ([30], [Th. 4.1]). Let the IVP (31) have a solution $y \in C[0, b]$, with $D^{\alpha} y \in C[0, b]$ and let $f \in C([0, b] \times \mathbb{R})$ such that its derivatives $\frac{\partial}{\partial t} f(t, y)$ and: $\frac{\partial^{2}}{\partial t^{2}} f(t, y)$ are continuous in $(0, b] \times \mathbb{R}$ and

$$
\left|\frac{\partial^{j}}{\partial y^{j}} f(t, y)\right| \leq \psi(|y|), \quad(t, x) \in(0, b] \times \mathbb{R}, j=0,1,2 .
$$


$\psi:[0, \infty) \rightarrow \mathbb{R}$ is a monotonically increasing function.

Then there exist $N_{0} \in \mathbb{N}$ and $\delta_{0}>0$ such that, for all $N \geq N_{0}$, the one-step collocation method possesses a unique solution $v \in S_{m-1}^{(-1)}\left(I_{N}\right)$ in the ball $\|u-z\|_{\infty} \leq \delta_{0}$, where $z=D^{\alpha} y \in C[0, b]$. If, in addition, the assumptions of Theorem 5 with $q:=m$ and $v \in[1-\alpha, 1)$ are fulfilled, then for all $N \geq N_{0}$, the following error estimate holds:

$$
\left\|y_{N}-y\right\|_{\infty} \leq c E_{N}(m, v, r)
$$

with $y_{N}$ given by Formula (39). Here $c$ is a constant not depending on $N$, and $E_{N}$ is defined by (40).

\subsection{Two-Step Collocation Methods for FDEs}

Given the graded mesh $I_{N}$ defined in (36) and collocation parameters $0 \leq \eta_{1}<\cdots<$ $\eta_{m} \leq 1$, with $\left(\eta_{1}, \eta_{m}\right) \neq(0,1)$, the two-step collocation method approximates the solution $z$ of (35) by a function $v \in S_{2 m-1}^{(-1)}\left(I_{N}\right)$. By defining the polynomial $v_{j}=\left.v\right|_{\left[t_{j-1}, t_{j}\right]}$, we impose these collocation and interpolation conditions, for $j=2, \ldots, N$ :

$$
\begin{gathered}
v_{j}\left(t_{j k}\right)=f\left(t_{j k},\left(J^{\alpha} v\right)\left(t_{j k}\right)+Q\left(t_{j k}\right)\right), \quad k=1, \ldots, m \\
v_{j}\left(t_{j-1, k}\right)=v_{j-1}\left(t_{j-1, k}\right), \quad k=1, \ldots, m
\end{gathered}
$$

The collocation solution $v(t)$ can be expressed as:

$$
v(t)=v_{1}(t)+\sum_{\lambda=2}^{N}\left(\sum_{k=1}^{m} z_{\lambda k} L_{\lambda, m+k}(t)+\sum_{k=1}^{m} z_{\lambda-1, k} L_{\lambda k}(t)\right), \quad t \in[0, T],
$$

where $v_{1}$ is obtained by a suitable starting procedure (cfr. [20]); $L_{\lambda, \mu}=L_{\mu}$ in $\left[t_{\lambda-1}, t_{\lambda}\right]$ and it is null outside. $L_{\mu}$ is the $\mu$-th Lagrange fundamental polynomial corresponding to the nodes $\left\{t_{j-1, k}, t_{j, k} \mid k=1, \ldots, m\right\}$. The coefficients $z_{\lambda \mu}$ are the solution of the nonlinear system:

$$
z_{j k}=f\left(t_{j k},\left(J^{\alpha} v\right)\left(t_{j k}\right)+Q\left(t_{j k}\right)\right), \quad k=1, \ldots, m .
$$

A more explicit formulation of the above system is:

$$
\begin{aligned}
z_{j k}=f\left(t_{j k},\left(J^{\alpha} v_{1}\right)\right. & \left(t_{j k}\right)+\sum_{\mu=1}^{m} z_{j \mu}\left(J^{\alpha} L_{j, m+\mu}\right)\left(t_{j k}\right)+\sum_{\lambda=2}^{j-1} \sum_{\mu=1}^{m} z_{\lambda \mu}\left(J^{\alpha} L_{\lambda, m+\mu}\right)\left(t_{j k}\right) \\
& \left.+\sum_{\mu=1}^{m} z_{j-1, \mu}\left(J^{\alpha} L_{j \mu}\right)\left(t_{j k}\right)+\sum_{\lambda=2}^{j-1} \sum_{\mu=1}^{m} z_{\lambda-1, \mu}\left(J^{\alpha} L_{\lambda \mu}\right)\left(t_{j k}\right)+Q\left(t_{j k}\right)\right),
\end{aligned}
$$

$k=1, \ldots, m$. Although a number of fractional integrals must be computed, they may be analytically evaluated, thus no further approximation is needed.

The main converge result is provided by the following theorem:

Theorem 7 ([20], [Th. 4.5]). Let hypothesis HP 1 of Theorem 6 hold. Then there exist $N_{0} \in \mathbb{N}$ and $\delta_{0}>0$ such that, for all $N \geq N_{0}$, the two-step collocation method possesses a unique solution $v \in S_{2 m-1}^{(-1)}\left(I_{N}\right)$ in the ball $\|u-z\|_{\infty} \leq \delta_{0}$, where $z=D^{\alpha} y \in C[0, T]$. If, in addition, the assumptions of Theorem 5 with $q:=2 m$ and $v \in[1-\alpha, 1)$ are satisfied, then for all $N \geq N_{0}$, the error is bounded as follows:

$$
\left\|y_{N}-y\right\|_{\infty} \leq c E_{N}(2 m, v, r),
$$

with $y_{N}$ defined in (39). Here, the value of the constant $c$ does not depend on $N$, and $E_{N}$ is defined in (40). 
It is evident that a suitable choice of the grading exponent $r$ is the basic step to obtain a high accuracy. The best choice of $r$ grows with $m$, i.e., with the number of collocation abscissae and also depends on the degree of smoothness of the analytical solution.

By comparing one- and two-step collocation methods, we observe that they have the same computational cost, since they both require the solution of a nonlinear system of dimension $m$, nevertheless, the error of the two-step method decreases as $O\left(N^{-2 m}\right)$, while the error of the one-step method decreases as $O\left(N^{-m}\right)$ (for both methods we considered the best case).

We provide a numerical illustration on the following test equation, taken from [61].

$$
\begin{array}{ll}
D^{\alpha} y(t)= & \frac{40320}{\Gamma(9-\alpha)} t^{8-\alpha}-3 \frac{\Gamma\left(5+\frac{\alpha}{2}\right)}{\Gamma\left(5-\frac{\alpha}{2}\right)} t^{4-\frac{\alpha}{2}}+\left(\frac{3}{2} t^{\frac{\alpha}{2}}-t^{4}\right)^{3} \\
& +\frac{9}{4} \Gamma(\alpha+1)-(y(t))^{\frac{3}{2}}, \\
y(0)=0, &
\end{array}
$$

$\alpha=1 / 2$. The exact solution is $y=t^{8}-3 t^{4+\alpha / 2}+\frac{9}{4} t^{\alpha}$. The hypotheses of Theorem 5 are satisfied by $v=0.5$ and any $q \in \mathbb{N}$. In Figure 2, we plot the work-precision diagram obtained by one- and two-step collocation methods, with collocation parameters equally spaced in $[0,1]$ with $\eta_{0} \neq 0$ and $\eta_{m} \neq 1$; with $r=\frac{m}{1-v}$ for the one-step methods and $r=\frac{2 m}{1-v}$ for the two-step methods. We observe that multivalue collocation obtain a definite improvement of one-step collocation methods, except for low accuracy requests.

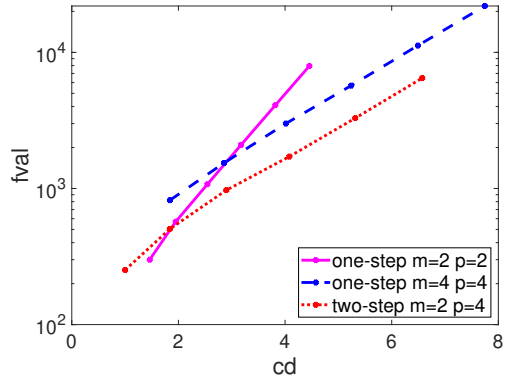

(a)

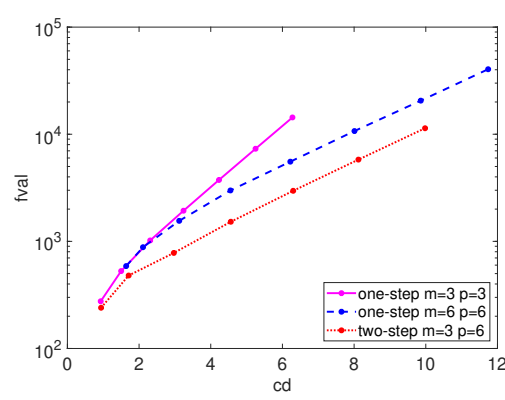

(b)

Figure 2. Work-precision diagrams for one- and two-step collocation methods for FDEs, $c d$ is the number of correct digits, and fval is the number of function evaluations. (a) One-step methods with $m=2$ and $m=4$, two-step methods with $m=2$; (b) one-step methods with $m=3$ and $m=6$, two step methods with $m=3$.

\section{Conclusions}

We presented a concise selection of our recent results on collocation methods for ODEs and FDE. This technique has exhibited a wide range of benefits in terms of accuracy and efficiency. Moreover, the choice of collocation basis makes the numerics more adapted to the problem, with meaningful improvements when qualitative behaviors of the solution are merged in the numerical scheme. Adapted functional basis are relevant, for instance, in the case of oscillatory problems [62-65]. Further developments of this research will be oriented to the establishment of a theory of collocation methods for stochastic problems (see, for instance, refs. [51,66-71] and references therein).

Author Contributions: All authors A.C., D.C., R.D., B.P. equally contributed to this work. All authors have read and agreed to the published version of the manuscript.

Funding: The authors are members of the INdAM Research group GNCS and are supported by the GNCS-INDAM project. A. Cardone, D. Conte, and R. D'Ambrosio are supported by PRIN2017-MIUR project 2017JYCLSF "Structure preserving approximation of evolutionary problems". 
Institutional Review Board Statement: Not applicable.

Informed Consent Statement: Not applicable.

Data Availability Statement: Not applicable.

Conflicts of Interest: The authors declare no conflict of interest.

\author{
Abbreviations \\ FDEs Fractional Differential Equation \\ GLM General Linear Method \\ IVP Initial Value Problem \\ ODE Ordinary Differential Equation \\ RK Runge-Kutta
}

The following abbreviations are used in this manuscript:

\title{
References
}

1. Butcher, J. Numerical Methods for Ordinary Differential Equations, 3rd ed.; John Wiley \& Sons, Inc.: Hoboken, NJ, USA, 2016.

2. Hairer, E.; Wanner, G. Solving Ordinary Differential Equations. II; Springer Series in Computational Mathematics, Stiff and Differential-Algebraic Problems, Second Revised Edition, Paperback; Springer: Berlin, Germany, 2010; Volume 14, p. xvi+614.

3. Lambert, J. Numerical Methods for Ordinary Differential Systems: The Initial Value Problem; John Wiley \& Sons, Inc.: Hoboken, NJ, USA, 1991.

4. D'Ambrosio, R.; Paternoster, B. Multivalue collocation methods free from order reduction. J. Comput. Appl. Math. 2021, 387, 112515. [CrossRef]

5. D'Ambrosio, R.; Ferro, M.; Jackiewicz, Z.; Paternoster, B. Two-step almost collocation methods for ordinary differential equations. Numer. Algorithms 2010, 53, 195-217. [CrossRef]

6. Costabile, F.; Gualtieri, M.; Napoli, A. Lidstone-based collocation splines for odd-order BVPs. Math. Comput. Simul. 2021, 186, 124-135. [CrossRef]

7. Costabile, F.; Napoli, A. Collocation for high order differential equations with two-points Hermite boundary conditions. Appl. Numer. Math. 2015, 87, 157-167. [CrossRef]

8. Costabile, F.; Napoli, A. Collocation for high-order differential equations with Lidstone boundary conditions. J. Appl. Math. 2012, 2012, 120792. [CrossRef]

9. Costabile, F.; Napoli, A. A class of collocation methods for numerical integration of initial value problems. J. Appl. Math. 2011, 62, 3221-3235. [CrossRef]

10. Costabile, F.; Napoli, A. Stability of Chebyshev collocation methods. Comput. Math. Appl. 2004, 47, 659-666. [CrossRef]

11. Lie, I. The stability function for multistep collocation methods. Numer. Math. 1990, 57, 779-787. [CrossRef]

12. Lie, I.; Nø rsett, S. Superconvergence for Multistep Collocation. Math. Comp. 1989, 52, 65-79. [CrossRef]

13. Blank, L. Stability of collocation for weakly singular Volterra equations. IMA J. Numer. Anal. 1995, 15, 357-375. [CrossRef]

14. Brunner, H. Cambridge monographs on applied and computational mathematics. In Collocation Methods for Volterra Integral and Related Functional Differential Equations; Cambridge University Press: Cambridge, UK, 2004; Volume 15, p. xiv+597.

15. Brunner, H.; Pedas, A.; Vainikko, G. Piecewise polynomial collocation methods for linear Volterra integro-differential equations with weakly singular kernels. SIAM J. Numer. Anal. 2001, 39, 957-982. [CrossRef]

16. Zayernouri, M.; Karniadakis, G.E. Fractional spectral collocation methods for linear and nonlinear variable order FPDEs. J. Comput. Phys. 2015, 293, 312-338. [CrossRef]

17. Cardone, A.; Conte, D. Multistep collocation methods for Volterra integro-differential equations. Appl. Math. Comput. 2013, 221,770-785. [CrossRef]

18. Cardone, A.; Conte, D.; Paternoster, B. A MATLAB Implementation of Spline Collocation Methods for Fractional Differential Equations. Lect. Notes Comput. Sci. 2021, 12949, 387-401.

19. Cardone, A.; Conte, D. Stability analysis of spline collocation methods for fractional differential equations. Math. Comput. Simulat. 2020, 178, 501-514.

20. Cardone, A.; Conte, D.; Paternoster, B. Two-step collocation methods for fractional differential equations. Discrete Contin. Dyn. Syst. Ser. B 2018, 23, 2709-2725. [CrossRef]

21. Conte, D.; D'Ambrosio, R.; Paternoster, B. Two-step diagonally-implicit collocation based methods for Volterra integral equations. Appl. Numer. Math. 2012, 62, 1312-1324.

22. Conte, D.; Paternoster, B. Multistep collocation methods for Volterra integral equations. Appl. Numer. Math. 2009, 59, 1721-1736.

23. Conte, D.; Jackiewicz, Z.; Paternoster, B. Two-step almost collocation methods for Volterra integral equations. Appl. Math. Comput. 2008, 204, 839-853. [CrossRef]

24. D'Ambrosio, R.; Paternoster, B. Two-step modified collocation methods with structured coefficient matrices. Appl. Numer. Math. 2012, 62, 1325-1334. 
25. Ford, N.; Morgado, M.; Rebelo, M. Nonpolynomial collocation approximation of solutions to fractional differential equations. Fract. Calc. Appl. Anal. 2013, 16, 874-891. [CrossRef]

26. Guo, B.Y.; Yan, J.P. Legendre-Gauss collocation method for initial value problems of second order ordinary differential equations. Appl. Numer. Math. 2009, 59, 1386-1408.

27. Guo, B.y.; Wang, Z.q. Legendre-Gauss collocation methods for ordinary differential equations. Adv. Comput. Math. 2009, 30, 249-280. [CrossRef]

28. Li, X. Numerical solution of fractional differential equations using cubic B-spline wavelet collocation method. Commun. Nonlinear Sci. Numer. Simul. 2012, 17, 3934-3946. [CrossRef]

29. Pedas, A.; Tamme, E. On the convergence of spline collocation methods for solving fractional differential equations. J. Comput. Appl. Math. 2011, 235, 3502-3514. [CrossRef]

30. Pedas, A.; Tamme, E. Numerical solution of nonlinear fractional differential equations by spline collocation methods. J. Comput. Appl. Math. 2014, 255, 216-230. [CrossRef]

31. Pedas, A.; Tamme, E. Spline collocation for nonlinear fractional boundary value problems. Appl. Math. Comput. $2014,244,502-513$. [CrossRef]

32. Wang, Z.Q.; Guo, B.Y. Legendre-Gauss-Radau collocation method for solving initial value problems of first order ordinary differential equations. J. Sci. Comput. 2012, 52, 226-255. [CrossRef]

33. Zayernouri, M.; Karniadakis, G.E. Fractional spectral collocation method. SIAM J. Sci. Comput. 2014, 36, A40-A62. [CrossRef]

34. Paternoster, B. Phase-fitted collocation-based Runge-Kutta-Nystrom method. Appl. Numer. Math. 2000, 35, 339-355. [CrossRef]

35. Conte, D.; D'Ambrosio, R.; D'Arienzo, M.P.; Paternoster, B. Multivalue mixed collocation methods. Appl. Math. Comput. 2021, 409, 126346. [CrossRef]

36. Norsett, S.; Wanner, G. Perturbed collocation and Runge Kutta methods. Numer. Math. 1981, 38, 193-208. [CrossRef]

37. Jackiewicz, Z. General Linear Methods for Ordinary Differential Equations; John Wiley \& Sons, Inc.: Hoboken, NJ, USA, 2009; p. xvi+482. [CrossRef]

38. Söderlind, G.; Jay, L.; Calvo, M. Stiffness 1952-2012: Sixty years in search of a definition. BIT Numer. Math. 2015, 55, 531-558. [CrossRef]

39. Cash, J. Efficient numerical method for the solution of stiff initial-value problems and differential algebraic equations. $R$. Soc. Lond. Proc. Ser. A Math. Phys. Eng. Sci. 2003, 459, 797-815. [CrossRef]

40. Bellomo, N.; Bingham, R.; Chaplain, M.A.; Dosi, G.; Forni, G.; Knopoff, D.A.; Lowengrub, J.; Twarock, R.; Virgillito, M.E. A multiscale model of virus pandemic: Heterogeneous interactive entities in a globally connected world. Math. Models Methods Appl. Sci. 2020, 30, 1591-1651. [CrossRef]

41. Heldt, F.; Frensing, T.; Pflugmacher, A.; Gröpler, R.; Peschel, B.; Reichl, U. Multiscale Modeling of Influenza A Virus Infection Supports the Development of Direct-Acting Antivirals. PLoS Comp. Biol. 2013, 9, e1003372. [CrossRef]

42. Southern, J.; Pitt-Francis, J.; Whiteley, J.; Stokeley, D.; Kobashi, H.; Nobes, R.; Kadooka, Y.; Gavaghan, D. Multi-scale computational modelling in biology and physiology. Prog. Biophys. Mol. Biol. 2008, 96, 60-89. [CrossRef] [PubMed]

43. Metzler, R.; Klafter, J. The random walk's guide to anomalous diffusion: A fractional dynamics approach. Phys. Rep. 2000, 339, 77. [CrossRef]

44. Mainardi, F. Fractional Calculus and Waves in Linear Viscoelasticity: An Introduction to Mathematical Models; Imperial College Press: London, UK, 2010; p. xx+347. [CrossRef]

45. Povstenko, Y. Thermoelasticity that uses fractional heat conduction equation. J. Math. Sci. 2009, 162, 296-305. [CrossRef]

46. Diethelm, K.; Ford, N.J.; Freed, A.D. A predictor-corrector approach for the numerical solution of fractional differential equations. Nonlinear Dynam. 2002, 29, 3-22. [CrossRef]

47. Lubich, C. Fractional linear multistep methods for Abel-Volterra integral equations of the second kind. Math. Comp. 1985, 45, 463-469. [CrossRef]

48. Garrappa, R.; Popolizio, M. On accurate product integration rules for linear fractional differential equations. J. Comput. Appl. Math. 2011, 235, 1085-1097. [CrossRef]

49. Khader, M.M. On the numerical solutions for the fractional diffusion equation. Commun. Nonlinear Sci. Numer. Simul. 2011, 16, 2535-2542. [CrossRef]

50. Burrage, K.; Cardone, A.; D’Ambrosio, R.; Paternoster, B. Numerical solution of time fractional diffusion systems. Appl. Numer. Math. 2017, 116, 82-94. [CrossRef]

51. Cardone, A.; D'Ambrosio, R.; Paternoster, B. A spectral method for stochastic fractional differential equations. Appl. Numer. Math. 2019, 139, 115-119. [CrossRef]

52. Blank, L. Numerical Treatment of Differential Equations of Fractional Order; Technical Report, Numerical Analysis Report; Department of Mathematics, University of Manchester: Manchester, UK, 1996.

53. Pedas, A.; Tamme, E. Spline collocation methods for linear multi-term fractional differential equations. J. Comput. Appl. Math. 2011, 236, 167-176. [CrossRef]

54. Cardone, A.; Conte, D.; Paternoster, B. Stability analysis of two-step spline collocation methods for fractional differential equations. submitted.

55. D'Ambrosio, R.; Hairer, E. Long-term stability of multi-value methods for ordinary differential equations. J. Sci. Comput. 2014, 60, 627-640. [CrossRef] 
56. D'Ambrosio, R.; Hairer, E.; Zbinden, C. G-symplecticity implies conjugate-symplecticity of the underlying one-step method. BIT Numer. Math. 2013, 53, 867-872. [CrossRef]

57. D'Ambrosio, R.; Esposito, E.; Paternoster, B. General linear methods for $y^{\prime \prime}=f(y(t))$. Numer. Algorithms 2012, 61, 331-349. [CrossRef]

58. Diethelm, K. An application-oriented exposition using differential operators of Caputo type. In The Analysis of Fractional Differential Equations; Lecture Notes in Mathematics; Springer: Berlin, Germany, 2010; Volume 2004, p. viii+247. [CrossRef]

59. Kilbas, A.A.; Srivastava, H.M.; Trujillo, J.J. Theory and Applications of Fractional Differential Equations; North-Holland Mathematics Studies; Elsevier Science B.V.: Amsterdam, The Netherlands, 2006; Volume 204, p. xvi+523.

60. Podlubny, I. Fractional Differential Equations; Mathematics in Science and Engineering; Academic Press, Inc.: San Diego, CA, USA, 1999; Volume 198, p. xxiv+340.

61. Diethelm, K.; Ford, N.J.; Freed, A.D. Detailed error analysis for a fractional Adams method. Numer. Algorithms 2004, 36, 31-52 [CrossRef]

62. Cardone, A.; D'Ambrosio, R.; Paternoster, B. Exponentially fitted IMEX methods for advection-diffusion problems. J. Comput. Appl. Math. 2017, 316, 100-108. [CrossRef]

63. Cardone, A.; D'Ambrosio, R.; Paternoster, B. High order exponentially fitted methods for Volterra integral equations with periodic solution. Appl. Numer. Math. 2017, 114, 18-29.

64. Cardone, A.; Ixaru, L.G.; Paternoster, B.; Santomauro, G. Ef-Gaussian direct quadrature methods for Volterra integral equations with periodic solution. Math. Comput. Simul. 2015, 110, 125-143. [CrossRef]

65. Ixaru, L.G.; Vanden Berghe, G. Exponential Fitting; Mathematics and its Applications, with 1 CD-ROM (Windows, Macintosh and UNIX); Kluwer Academic Publishers: Dordrecht, The Netherlands, 2004; Volume 568, p. xiv+308.

66. D'Ambrosio, R.; Giordano, G.; Paternoster, B.; Ventola, A. Perturbative analysis of stochastic Hamiltonian problems under time discretizations. Appl. Math. Lett. 2021, 409, 107223. [CrossRef]

67. D'Ambrosio, R.; Di Giovacchino, S. Mean-square contractivity of stochastic theta-methods. Comm. Nonlin. Sci. Numer. Simul. 2021, 96, 105671. [CrossRef]

68. D'Ambrosio, R.; Di Giovacchino, S. Nonlinear stability issues for stochastic Runge-Kutta methods. Comm. Nonlin. Sci. Numer. Simul. 2021, 94, 105549. [CrossRef]

69. Conte, D.; D'Ambrosio, R.; Paternoster, B. Improved theta-methods for stochastic Volterra integral equations. Comm. Nonlin. Sci. Numer. Simul. 2021, 93, 105528. [CrossRef]

70. D'Ambrosio, R.; Scalone, C. On the numerical structure preservation of nonlinear damped stochastic oscillators. Numer. Algorithms 2021, 86, 933-952. [CrossRef]

71. Conte, D.; D'Ambrosio, R.; Giordano, G.; Paternoster, B. Continuous Extension of Euler-Maruyama Method for Stochastic Differential Equations. Lect. Notes Comput. Sci. 2021, 12949, 135-145. 